1997-11-01

\title{
Extracting the Relative Grain Boundary Free Energy and Mobility Functions from the Geometry of Microstructures
}

\author{
Brent L. Adams \\ b_I_adams@byu.edu \\ D. Kinderlehrer \\ W. W. Mullins \\ Anthony D. Rollett \\ Shlomo Ta'asan
}

Follow this and additional works at: https://scholarsarchive.byu.edu/facpub

Part of the Mechanical Engineering Commons

\section{Original Publication Citation}

Scripta Materialia, Vol. 38, No. 4, pp. 531-536, 1998

\section{BYU ScholarsArchive Citation}

Adams, Brent L.; Kinderlehrer, D.; Mullins, W. W.; Rollett, Anthony D.; and Ta'asan, Shlomo, "Extracting the Relative Grain Boundary Free Energy and Mobility Functions from the Geometry of Microstructures" (1997). Faculty Publications. 658.

https://scholarsarchive.byu.edu/facpub/658

This Peer-Reviewed Article is brought to you for free and open access by BYU ScholarsArchive. It has been accepted for inclusion in Faculty Publications by an authorized administrator of BYU ScholarsArchive. For more information, please contact ellen_amatangelo@byu.edu. 


\title{
EXTRACTING THE RELATIVE GRAIN BOUNDARY FREE ENERGY AND MOBILITY FUNCTIONS FROM THE GEOMETRY OF MICROSTRUCTURES
}

\author{
B.L. Adams*, D. Kinderlehrer**, W.W. Mullins*, A.D. Rollett* \\ and Shlomo Ta'asan** \\ *Materials Science and Engineering, Carnegie Mellon University, Pittsburgh, PA 15213-3890 \\ **Mathematical Sciences, Carnegie Mellon University, Pittsburgh, PA 15213-3890 \\ http://www.sciencedirect.com/science/journal/13596462
}

\begin{abstract}
1. Introduction
The local physio-chemical characteristics of grain boundaries are known to depend strongly upon the crystallographic and thermodynamic variables $(1,2)$. The way in which the corresponding anisotropy is distributed through the network of grain boundaries influences many important macroscopic properties such as the propagation of corrosion and fracture (3). It is also a key factor in the evolution of microstructures through a complex interplay of the thermodynamic driving forces and kinetic responses which determine the motions of grain boundaries. Hence the importance of a knowledge of the orientation dependent properties of grain boundaries.

This paper describes a method for extracting, from measurements of a polycrystal, the relative excess free energy and the relative mobility of the grain boundaries as functions of the crystallographic type (five degrees of freedom) and relevant thermodynamic variables. The method requires the simultaneous measurement of both the geometry and the crystallography of a large number of grain boundary intersections; the crystallographic information may be obtained from orientation imaging microscopy (OIM) (4). For simplicity, the intersections will be assumed to be triple junctions (three intersecting boundaries). The energies and mobilities are obtained as ratios to respective standards that must be determined independently.

The determination of grain boundary energies is based on the assumption of local equilibrium at the triple junctions; thus each triple junction is assumed to obey the well known Herring equilibrium equations (5). The assumption can be checked from consistency of the data. The novel contribution we offer is a new mathematical method for extracting the grain boundary free energy as a function of its crystallographic type from a vast number of these equations. This is not a trivial problem because, in general, the equation for any given triple junction connects the free energies (and their derivatives) at widely separated values of the independent variables. Straightforward consecutive solution of these equations can result in large instabilities. The new method obviates this problem.

The relative mobilities of the boundaries meeting at a triple junction will be shown to satisfy an equation of constraint, assuming that the local grain boundary velocity is proportional to the local thermodynamic driving force (i.e. curvature motion). A large number of such equations, one for each triple junction, may be treated by the same method used in the energy case resulting in the relative
\end{abstract}




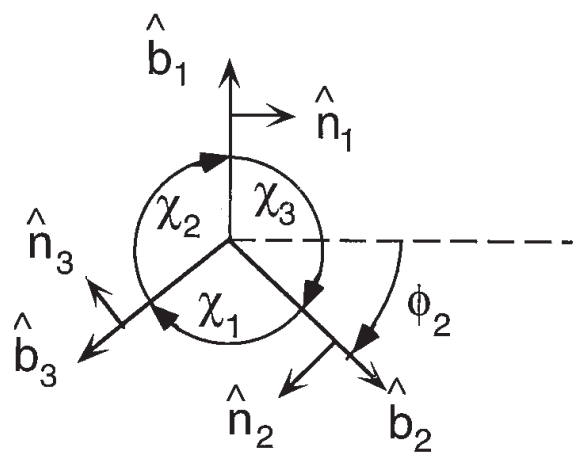

Figure 1. Definition of geometric parameters associated with boundaries adjoining a triple junction. Each boundary is associated with a (unit) normal, $\mathbf{n}$, a (unit) direction, $\mathbf{b}$, an inclination, $\phi$, and a dihedral angle, $\chi$. Only one inclination angle $\left(\phi_{2}\right)$ is labeled for clarity.

mobility as a function of the grain boundary type (and thermodynamic variables). It is remarkable that (relative) mobilities may be inferred from the microstructure at a given instant; evolution data are required only to establish the absolute mobility of a standard comprising the motion of a particular grain boundary under a known driving force.

In section 2, we review Herring's equation relating the grain boundary energies at a triple junction to the intersection angles. In section 3 we present the equation connecting the mobilities. In section 4 , the mathematical method will be illustrated for the energy case, using the simplifying assumption that the energy is independent of the grain boundary inclination and that the misorientation can be specified by one angle as in a fiber texture. The illustration comprises $1.3 \times 10^{5}$ randomly generated triple junctions with an assumed hypothetical free energy function.

\section{Equilibrium Thermodynamics Of Triple Junctions}

Triple junctions are presumed to be in local equilibrium so that they obey the Herring equations which may be written for the triple junction depicted in Fig. 1 as

$$
\sum_{j=1}^{3}\left\{\sigma_{\mathrm{j}} \hat{\mathrm{b}}_{\mathrm{j}}+\left(\partial \sigma_{\mathrm{j}} / \partial \phi_{\mathrm{j}}\right) \hat{\mathrm{n}}_{\mathrm{j}}\right\}=\overrightarrow{0}
$$

Here $\sigma_{\mathrm{j}}$ is the excess free energy of the $\mathrm{jth}$ boundary, $\hat{\mathrm{n}}_{\mathrm{j}}$ is the unit boundary normal of the jth boundary, $\hat{b}_{j}$ is a unit vector lying in the jth boundary and perpendicular to the triple line $\hat{l}=\hat{n}_{j} \otimes \hat{b}_{j}$ which is common to all three adjacent boundaries, and $\phi_{\mathrm{j}}$ is defined to be the right-handed angle of rotation about $\hat{I}$ of the jth boundary from a reference direction. If any boundary is in an energy cusp orientation, special care must be taken in defining the corresponding torque term in Eq.(1) $(5,7)$.

\section{Relationship Among Boundary Mobilities at a Triple Junction}

Grain boundary velocities at a triple junction are related since the motion of any two of the intersecting boundaries defines a new locus of intersection to which the third boundary must move if the triple junction is to remain intact. The equation connecting the velocities becomes an equation connecting the 
mobilities when the driving forces are specified; the latter require knowledge of the local curvature and energy function of each grain boundary at the triple junction.

To derive the equation connecting velocities, let $\vec{V}$ be the velocity of the triple junction in the direction normal to the triple junction line, and $v_{i}$ be the velocity of the ith attached grain boundary in the direction of its normal. Then if the triple junction remains intact, the relation

$$
v_{i}=\vec{V} \cdot \hat{n}_{i}
$$

must be satisfied, where $\hat{n}_{i}$ is the corresponding normal; here the grain boundary normals are chosen in the same sense around the triple junction as shown in Fig. 1. The normals are linearly dependent, however, since any one can be expressed in terms of the other two. Use of cross products establishes that the relation among them is

$$
\sum_{i} \sin \left(\chi_{\mathrm{i}}\right) \hat{n}_{\mathrm{i}}=0
$$

Therefore, multiplying Eq.(2) by $\sin \left(\chi_{\mathrm{i}}\right)$ and summing on $\mathrm{i}$ gives

$$
\sum_{i} \sin \left(\chi_{i}\right) v_{i}=0
$$

as the relation among the normal velocities at the triple junction. Finally, if we assume these velocities are given by $v_{i}=m_{i} f_{i}$, where $m_{i}$ is the mobility and $f_{i}$ is the local driving force, then

$$
\sum_{i}\left\{\sin \left(\chi_{i}\right) f_{i}\right\} m_{i}=0
$$

if the coefficients of the $m_{i}$ (i.e. the products $\sin \left(\chi_{i}\right) f_{i}$ ) are known, then Eq. (5) connects the unknown mobilities. But the coefficients are determined by the angles of intersection $\chi_{\mathrm{i}}$ and by the driving forces given by the Herring formula (5),

$$
\mathrm{f}=\left(\sigma+\sigma_{\theta_{1} \theta_{1}}\right) \kappa_{1}+\left(\sigma+\sigma_{\theta_{2} \theta_{2}}\right) \kappa_{2}
$$

in which $\kappa_{1}$ and $\kappa_{2}$ are the principal curvatures, and $\sigma_{\theta_{1} \theta_{1}}$ and $\sigma_{\theta_{2} \theta_{2}}$ are angular derivatives of $\sigma$ along the principal curves; hence the $f_{i}$ are determined by the curvatures and energy functions of the boundaries at the triple junctions. Given these quantities, the coefficients of Eq. (5) are determined and the equation represents one relation among the unknown mobilities for each triple junction.

Special cases of Eq. (5) have been derived before. For uniform grain boundaries, $\chi_{\mathrm{i}}=120^{\circ}$ and $\mathrm{f}_{\mathrm{i}}=$ $\sigma \kappa_{\mathrm{i}}$ where $\kappa_{\mathrm{i}}$ is the curvature of the $\mathrm{i}^{\text {th }}$ boundary, Eq. (5) then states that the sum of the curvatures at the vertex must be zero. This is identical to the result of Weaire (8). For boundaries whose energies may depend on misorientation (but not on inclination), we may write $f_{i}=\sigma_{i} / R_{i}$ where $R_{i}$ refers to the radius of curvature of the $i^{\text {th }}$ boundary. The equation then is closely related to Eq. (6) of Maurice and Humphreys (9) but differs in the following respect: their equation appears in the context of a discussion of the vertex model of grain growth in which the grain boundaries are assumed to be arcs of circles between triple junctions; the $\mathrm{R}_{\mathrm{i}}$ are radii of curvature of these arcs. Our Eq. (5) is independent of any assumption about the geometry of the boundaries; $\mathrm{f}_{\mathrm{i}}$ and the associated curvatures are to be evaluated at the triple junction, i.e. in a strictly local sense.

In a multiple junction, consisting of the intersection of $n$ grain boundaries that remain attached as the intersection moves, any two boundaries may be selected and these together with each of the remaining $\mathrm{n}-2$ boundaries in turn must satisfy Eq. (5), leading to $\mathrm{n}-2$ independent equations among the mobilities at that junction. 


\section{A New Statistical Multiscale Method for the Extraction of the Free-Energy Function}

We limit the discussion to the proposed method of extraction of the free energy function from triple junction measurements; the same procedure can be used to extract the mobility function. The only difference is that two independent relations among energies are available at each triple junction whereas only one relation among mobilities is available.

To illustrate the new method, we restrict our attention to microstructures with a fiber texture so that crystallographic orientation is specified by a single angle about the fiber axis and further assume that the energy, $\sigma$, is a function of lattice misorientation, $\Delta \mathrm{g}$, alone, i.e. torque terms may be neglected. Under these circumstances, Eq. (1) reduces to the force balance consisting of three (Young's) relations (10)

$$
\frac{\sigma_{1}}{\sin \chi_{1}}=\frac{\sigma_{2}}{\sin \chi_{2}}=\frac{\sigma_{3}}{\sin \chi_{3}}
$$

where each equation involves two $\sigma$ 's and hence two values of $\Delta \mathrm{g}$. The objective is to find $\sigma$ as a function of $\Delta \mathrm{g}$ given the values $\Delta \mathrm{g}$ and $\chi$ for a vast number of triple junctions. We propose a method that is statistical and whose implementation is multiscale. In this way, we shall focus implicitly on the distributions of the quantities we are seeking. The issues that concern us here do not fall within the usual framework of least squares methods because, as shown below, it is the coefficient matrix in the equation which contains the experimental information rather than its right hand side.

To begin, discretize the range of $\Delta \mathrm{g}$ into $\mathrm{N}$ subintervals, say, $[0,1 / \mathrm{N} \pi),[1 / \mathrm{N} \pi, 2 / \mathrm{N} \pi), \ldots$, $[\mathrm{N}-1 / \mathrm{N} \pi, \pi]$, and also tabulate the equations. The values $\left(\Delta \mathrm{g}_{1}, \Delta \mathrm{g}_{2}\right)$ occurring in a given relation in Eq. (7) typically fall into two of these intervals. Establish $\left(\begin{array}{l}N \\ 2\end{array}\right)=\mathrm{N}(\mathrm{N}-1) / 2$ urns for the pairs $\left(\Delta \mathrm{g}_{1}, \Delta \mathrm{g}_{2}\right)$ and place each equation into its respective urn, imposing the statistical hypothesis that each such placement is an independent trial of identically distributed random pairs. We now exploit the Law of Large Numbers which tells us that the running average of the coefficients is normally distributed. This then permits us to average the equations in each urn to obtain a set of $\left(\begin{array}{l}N \\ 2\end{array}\right)$ averaged equations. The objective of this averaging or coarse graining procedure is to re-cast the problem in a robust form suitable for solution. The matrix of coefficients, $\mathrm{A}_{\mathrm{ij}}$, in Eq. (8) then contains the averaged relations between energies, $\sigma_{\mathrm{j}}$ is a vector of the energy function to be determined and $b_{i}$ is a zero vector.

$$
\sum_{1}^{\mathrm{N}} \mathrm{A}_{\mathrm{ij}} \sigma_{\mathrm{j}}=\mathrm{b}_{\mathrm{i}}, \quad \mathrm{i}=1, \ldots, \mathrm{N}(\mathrm{N}-1) / 2
$$

If $\mathrm{N}$ is large, e.g., $\mathrm{N}=256$, there obtains 32,896 equations, still a large number for numerical resolution. Thus it is useful to introduce a multiscale iterative method. Begin with a small $\mathrm{N}$ and solve the small system for a trial energy, say $\sigma^{\mathrm{N}}$. $\mathrm{N}$ is then increased to $2 \mathrm{~N}$, the data set is resorted and averaged, and resolution at level $2 \mathrm{~N}$ is initiated with the solution $\sigma^{\mathrm{N}}$ of the previous level. It turns out that by adapting a Kaczmarz method, the sparseness of the coefficient matrix is preserved and a solution found rapidly.

Figure 2 shows the result of this iteration for a trial simulation consisting of $1.3 \times 10^{5}$ randomly generated triple junctions or $3.9 \times 10^{5}$ equations. This simulation, accurate to within $0.5 \%$, required less than 4 minutes on a workstation. The trial function

$$
\sigma(\Delta \mathrm{g})=2+0.3 \sin 4 \Delta \mathrm{gl}
$$




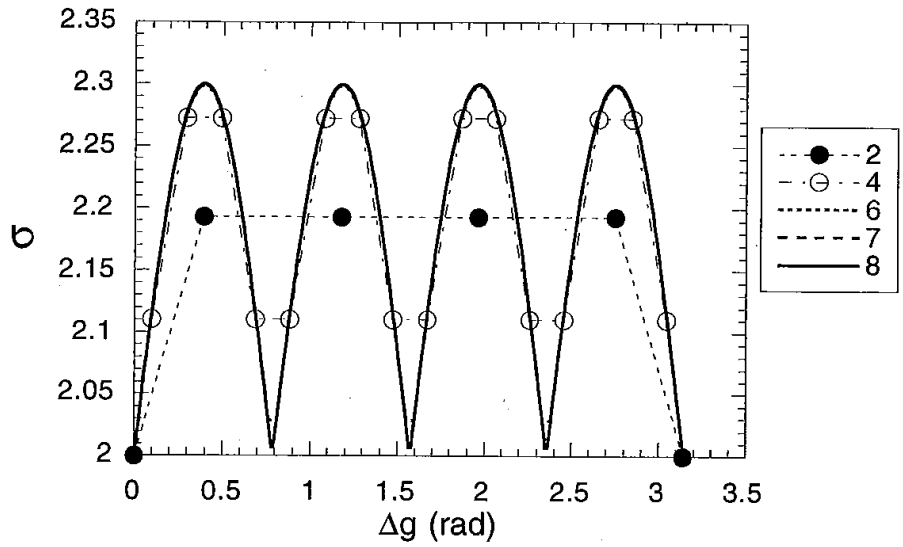

Figure 2. Reconstruction of the free energy function, $\sigma$, based on a simulated set of triple junction angles derived from a trigonometric function, showing five of the eight successive orders of approximation, beginning with $\mathrm{N}=2$ and ending with $\mathrm{N}=$ 256. The number of points is two raised to the power of the order. Orders $2(N=4)$ and $4(N=4)$ are shown with triangular and round symbols, respectively, to emphasize the result of the early stages of approximation, whereas stages 6,7 and $8(\mathrm{~N}=$ 64,128 , and 256) are indistinguishable on this plot, owing to the rapid convergence on the energy function on the trial function (Eq. 9).

was used to calculate sets of triple junction. Figure 3 shows that the error decreases monotonically with each successive refinement of the fitting of the energy function.

\section{Summary Remarks}

We have discussed a method of extracting the relative free energy and mobility of grain boundaries as functions of the grain boundary type (crystallographic variables of misorientation and inclination) and thermodynamic variables from the measurement of a vast number of triple junctions. Each triple junction, assumed locally equilibrated, provides three Herring equations (5) for the free energy function

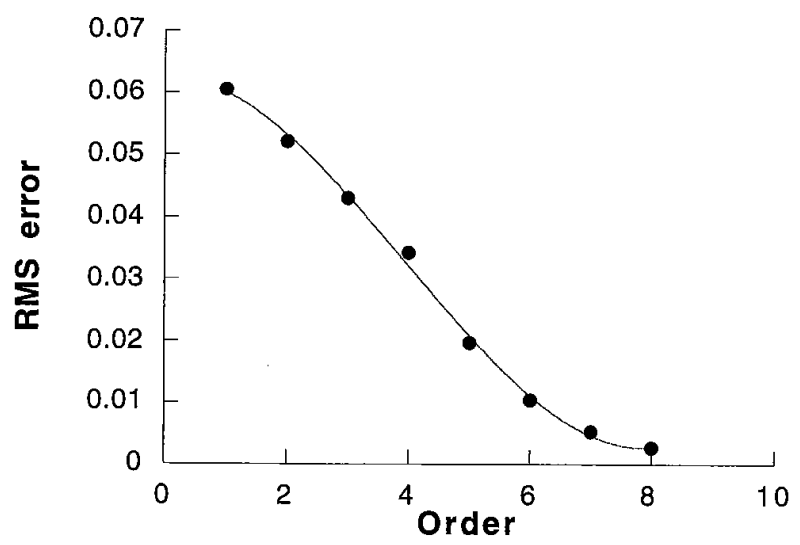

Figure 3. Root mean square error at each stage of the reconstruction of the free energy function, based on a simulated set of triple junction angles derived from a trigonometric function given in the text. The smooth line fitted through the points has been added to guide the eye. 
and one equation of the form of Eq. (5) (newly formulated) for the mobility; the latter relation assumes only that the local velocity of grain boundaries is proportional to the local capillary driving force. A new statistical/multiscale method was described to extract the free energy and mobility functions from the vast number of triple junction equations. The convergence of the method was illustrated for an example of data from randomly generated triple junctions based on a hypothetical free energy function.

Orientation imaging microscopy, when coupled with precision serial polishing, is now capable of revealing the 3 -dimensional structure of polycrystals to resolutions of about $100 \mathrm{~nm}$ (3). When the temperatures and times of equilibration have been sufficient, the mesostructure revealed by this tomography can be expected to satisfy Herring's force and torque balance relations at triple junctions as well as the mobility relation described by Eq. (5). Thus, the mesostructure carries data required to extract a mapping relating both the relative grain boundary excess free energy and the relative mobility to the character of the grain boundaries over the full fundamental zone. This approach defines a new inverse problem in materials science.

\section{$\underline{\text { Acknowledgment }}$}

This work was supported primarily by the MRSEC Program of the National Science Foundation under Award Number DMR-9632556.

\section{$\underline{\text { References }}$}

1. P. Lejcek and S. Hofmann, CRC Critical Reviews in Solid State and Materials Science. 20, 1 (1995).

2. G. Gottstein and F. Schwarzer, Mat. Sci. Forum. 94-96, 187-208 (1992).

3. T. Pan, B. L. Adams, T. Olson, and N. Panayotou, Acta mater. 44, 4685-4695 (1996).

4. B. L. Adams, S. I. Wright, and K. Kunze, Met. Trans. 24A, 819 (1993).

5. C. Herring, in Structure and Properties of Solid Surfaces, ed. R. Gomer and C. S. Smith, University of Chicago Press, Chicago (1952).

6. B. Adams, D. Kinderlehrer, S. Ta'asan, W. W. Mullins, S. Costiner, in Proc. MRS Symp. on Polycrystalline Thin Films III, San Francisco, in press (1997).

7. W. W. Mullins, in Metal Surfaces: Structure, Energetics and Kinetics, ed. W. D. Robertson and N. A. Gjostein, p. 17, The American Society for Metals, Ohio (1963).

8. D. Weaire, Philos. Mag. Lett. 68, 93-98 (1993).

9. Cl. Maurice and F. J. Humphreys, in Thermomechanical Processing: Theory, Modelling and Practice, ASM Intl., Ohio, in press (1997).

10. C. S. Smith, Trans. AIME. 175, 15 (1948). 